\title{
Lifetimes of the lowest triplet state of ozone by intracavity laser absorption spectroscopy
}

\author{
Bernd Abel ${ }^{a, b}$, Aleš Charvát ${ }^{a, b}$, Sabine F. Deppe ${ }^{a, b}$ \\ "Abteilung Spektroskopie und Photochemische Kinetik, Max-Planck Institut für biophysikalische Chemie, Am Faßberg 11, 37077 Göttingen, \\ Germany \\ ${ }^{\mathrm{b}}$ Institut für Physikalische Chemie der Universität Göttingen, Tammannstr. 6, 37077 Göttingen, Germany
}

Received 10 June 1997; in final form 5 August 1997

\begin{abstract}
High resolution spectra $\left(0.014 \mathrm{~cm}^{-1}\right)$ of $\mathrm{O}_{3}$ of the highly structured first two members of the Wulf progression in the near-infrared spectral range $\left(9100-10200 \mathrm{~cm}^{-1}\right)$ have been measured by intracavity laser absorption spectroscopy (ICLAS). A preliminary analysis of the rotational structure confirmed previous assignments for the lowest excited ${ }^{3} \mathrm{~A}_{2}$ (triplet) state. However, the linewidths of the ${ }^{3} \mathrm{~A}_{2} \leftarrow{ }^{1} \mathrm{~A}_{1}$ transitions have been found to be considerably broadend depending upon the $J$ and $K$ rotational quantum number, as well vibrational excitation. The short lifetimes inferred from these linewidths suggest that the rovibrational states of ozone's lowest triplet state are predissociative and not "metastable". (C) 1997 Elsevier Science B.V.
\end{abstract}

\section{Introduction}

The spectroscopy of ozone has attracted many scientists during the last half century and more, because of the importance of this molecule in controlling the thermal and radiation budgets as well as the chemistry of the Earth's atmosphere [1]. The Wulf optical absorption bands of $\mathrm{O}_{3}$ are a series of weak progressions partially diffuse starting just above the $\mathrm{O}_{2}+\mathrm{O}$ dissociation threshold. In the literature they are often trivially denoted as " $0-0$ " and " $1-0$ Wulf bands", which has only little meaning but was due to the fact that the assignments were not known until just recently. Wulf himself observed the diffuse absorption bands in the visible and near-infrared, respectively, several decades ago but made no electronic state assignments to the data [2]. However, since they have been observed, these bands are of great interest to those studying ozone chemistry and kinetics, since they provide a possibility of investigating and identifying the electronically excited states of ozone near the dissociation threshold and their potential impact on ozone's recombination and photodissociation dynamics in the near IR [1]. While the diffuse Hartley-, Huggins-, and Chappuis-bands in the UV/VIS spectral range can be attributed to singlet-singlet transitions [1], theoretical efforts by Banichevich et al. [3,4], Braunstein et al. [5-7], and Minaev et al. [8] indicate that the weak Wulf absorption band results from singlet-triplet transitions from the ${ }^{1} A_{1}$ ground state to one or more of the triplet states ${ }^{3} \mathrm{~A}_{2},{ }^{3} \mathrm{~B}_{2}$, and ${ }^{3} \mathrm{~B}_{1}$ (in $\mathrm{C}_{2 \mathrm{v}}$ symmetry and in the accepted energetic order $T_{0}\left({ }^{3} \mathrm{~A}_{2}\right)<T_{0}\left({ }^{3} \mathrm{~B}_{2}\right)<$ $\left.T_{0}\left({ }^{3} \mathrm{~B}_{1}\right)\right)$. Of these the most likely (strongest) transition being responsible for the Wulf bands is the ${ }^{3} \mathrm{~A}_{2} \leftarrow{ }^{\mathrm{I}} \mathrm{A}_{1}$ transition which becomes allowed through 
a)
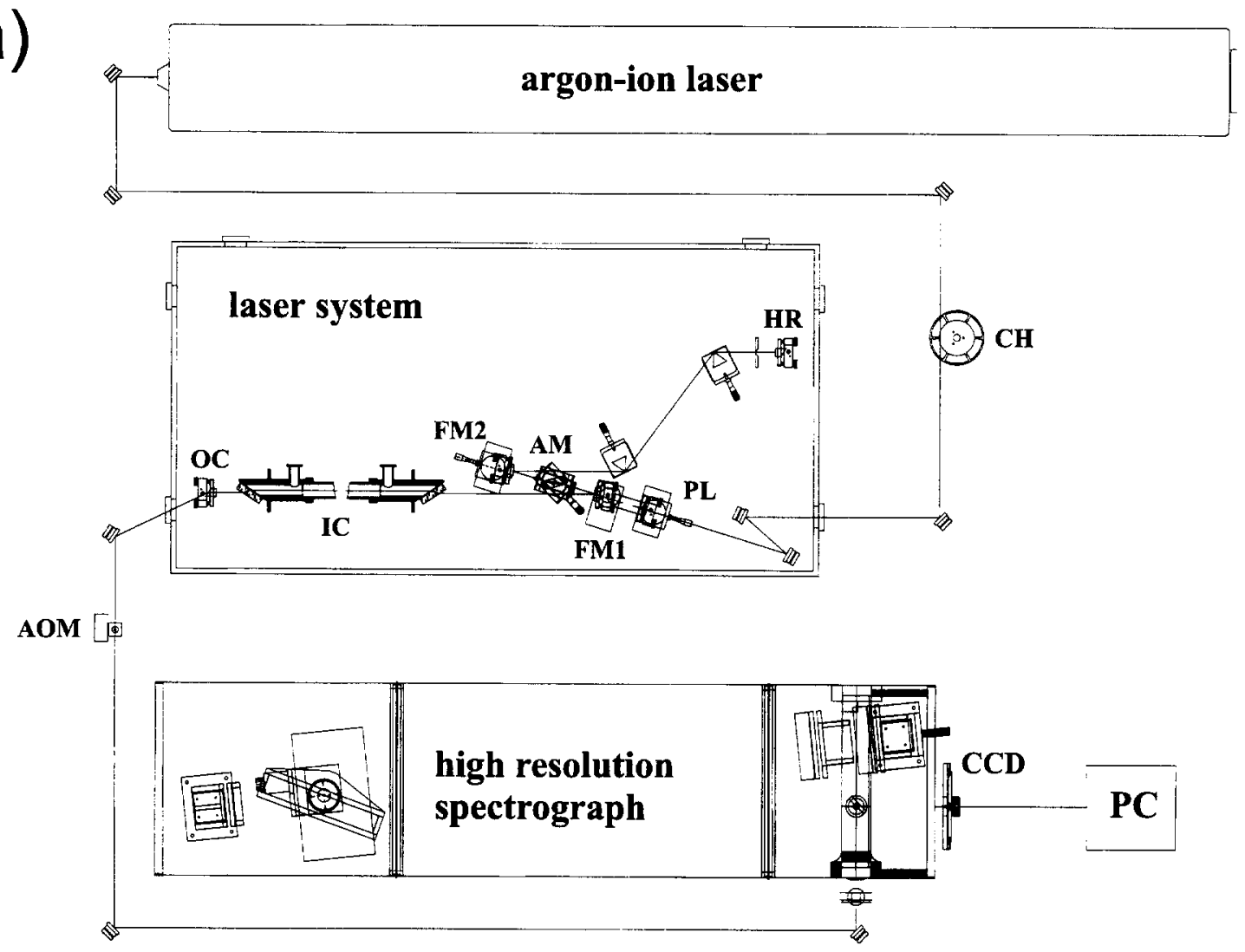

b)

\section{experimental resolution}
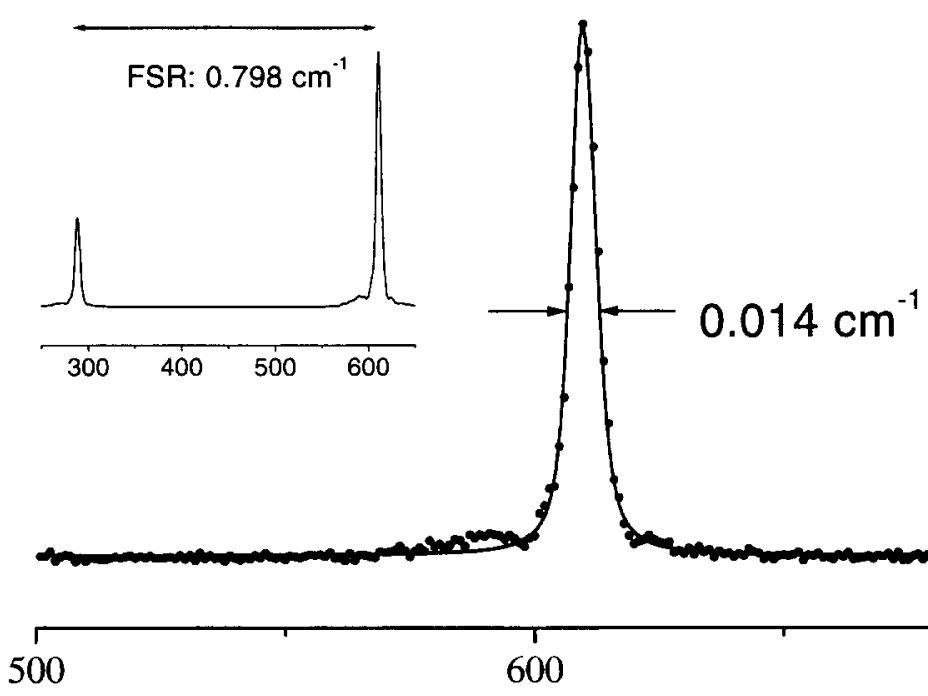

700

Pixel number 
spin-orbit coupling to the ${ }^{1} B_{2}$ state [8]. A set of pioneering experimental studies from Anderson and co-workers $[9,10]$ has provided further insight into the assignments of the ozone spectrum in the red and near infrared spectral range. Using optical absorption spectroscopy and isotopic substitution they were able to observe transitions to all three low-lying electronic triplet states of ozone and in turn to identify the lowest triplet states of ozone to be ${ }^{3} \mathrm{~A}_{2},{ }^{3} \mathrm{~B}_{2}$, and ${ }^{3} \mathrm{~B}_{1}$. They have tentatively assigned the ${ }^{3} \mathrm{~A}_{2}$ to be the upper state in the spectra in the spectral range near 1 $\mu \mathrm{m}$ and in turn found the ${ }^{3} \mathrm{~A}_{2} \leftarrow{ }^{1} \mathrm{~A}_{1}$ transition to have the largest intensity whereas transitions to the other triplet states are hardly visible in absorption (only as small shoulders in the relatively strong ${ }^{3} \mathrm{~A}_{2} \leftarrow{ }^{1} \mathrm{~A}_{1}$ transition). However, from the determination of the origins of the bands the energetic position and order of the triplet states have been inferred. Without being able to fully resolve the rotational structure in the bands their assignments of the first two bands of the Wulf progression (which turned out to be a progression in the bending mode of $\mathrm{O}_{3}$ ) have been ${ }^{3} \mathrm{~A}_{2}(000) \leftarrow{ }^{1} \mathrm{~A}_{1}(000)$ and ${ }^{3} \mathrm{~A}_{2} \quad(010) \leftarrow 1$ $A_{1}(000)$. The ${ }^{3} A_{2}(020) \leftarrow{ }^{1} A_{1}(000)$ band has been found to be diffuse [9] which was interpreted to be an evidence for a barrier in the excited state. Just very recently Bouvier et al. [11] used high resolution FTIR absorption spectroscopy to record the two bands with a higher resolution. They confirmed the upper state assignments of Anderson et al. [9] and made preliminary assignments in the least perturbed and congested regions of the complicated rotationally resolved spectra. From their spectroscopic results on the first members of the Wulf progression they concluded that the lowest ${ }^{3} \mathrm{~A}_{2}$ state is metastable with a radiative lifetime of $0.01-0.1 \mathrm{~s}$ [11]. Several techniques other than photoabsorption have been used to study $\mathrm{O}_{3}$ in the near-IR region, some of which have lead researchers to conclude that bound excited states exist below the ground state dissociation limit [1214]. Swanson and Celotta [15] studied ozone using electron energy loss spectroscopy and concluded that bound energy levels of the ${ }^{3} B_{2}$ state exist. Recently, Neumark and co-workers [16] used photoelectron spectroscopy of the $\mathrm{O}_{3}^{-}$ion and accessed all electronically excited triplet states spectroscopically, and re-examined their energetic order. All electronically excited triplet states were found to lie above the ground state disscociation limit with energetic positions in good agreement with Anderson et al. [9]. In addition, many kinetic experiments have been reported which provided indirect "evidence" for the existence of metastable states e.g., $[17,18]$. The discussion about the metastable electronically excited triplet states and the presence of rotational structure in the Wulf band of $\mathrm{O}_{3}$ encouraged us to record high resolution absorption spectra of the first members of the Wulf band. From a systematic investigation of the spectral linewidths and the nature of the excited state(s) we expected to address the question of metastability of optically accessible triplet states of ozone. In order to avoid confusion. we would like to point out that our interpretation of "metastability" is connected with the overall lifetimes of the molecules quantum states. Therefore, from our point of view only stable bound states with (long) lifetimes (which are limited by spontaneous emission) are considered to be metastable and molecules with very short lifetimes are not.

\section{Experimental}

The experimental setup and the experimentally determined apparatus function of the spectrometer is depicted in Fig. 1a, b. Since details have already been given elsewhere [19], only a short description and the specifics of the present setup relevant to the present results will be given here. Briefly, the apparatus employed in these experiments is a solid-state intracavity laser absorption spectrometer (ICLAS) with two types of amplifying media, Ti:sapphire

Fig. 1. (a) Solid-state intracavity laser absorption spectrometer setup. AOM: opto acoustic modulator; CH: mechanical chopper; CCD: CCD-array; OC: output coupler; IC: intracavity cell; FMI(2): folding mirrors; AM: amplifying medium; HR: high reflector, PL: focussing lens for pump beam. (b) Experimental resolution of the ICLAS system $\left(0.014 \mathrm{~cm}^{-1}\right)$ measured with an etalon (free spectral range. FSR, $\left.=0.798 \mathrm{~cm}^{-1}\right)$. The etalon trace @ $9447 \mathrm{~cm}^{-1}(1058.5 \mathrm{~nm})$ has been fitted with a Lorentz profile in order to determine the apparatus function of the laser system. 
crystal (e.g., [19]) and Nd:glass [20], operating in the wavelength region between $700-1000 \mathrm{~nm}$, and beyond $1 \mu \mathrm{m}$, respectively. The laser spectrometer in an astigmatism compensated linear $Z$ configuation with two intracavity prisms for tuning were pumped by $2-5 \mathrm{~W}$ (multiline operation) of an argon ion laser

a)

$$
K^{\prime \prime}=12
$$

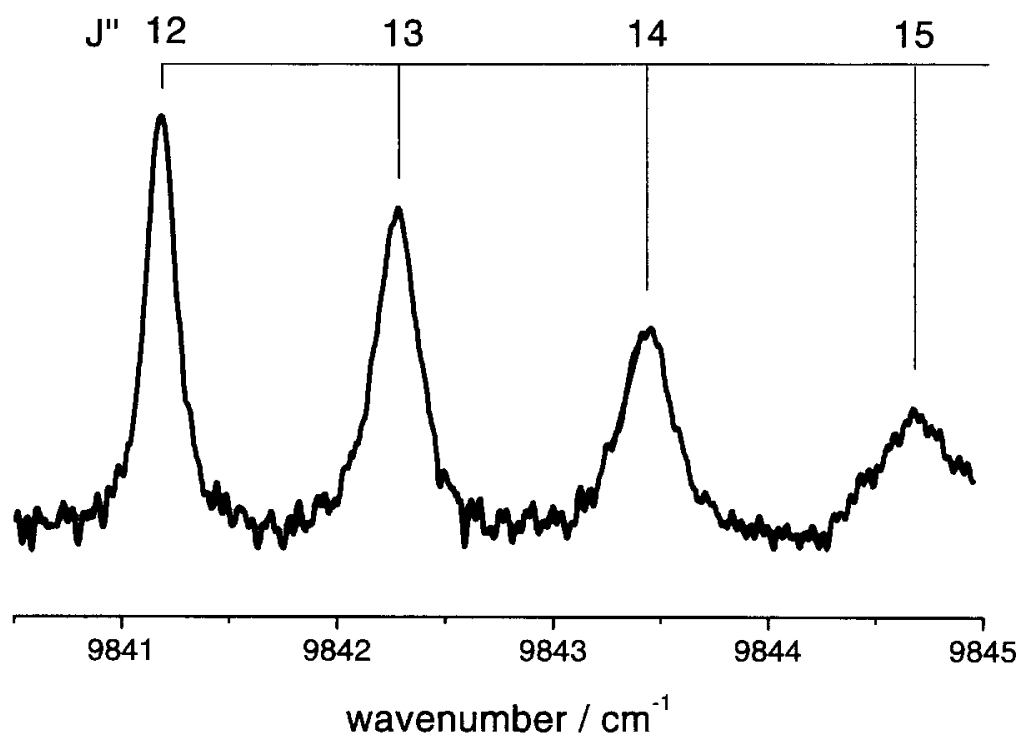

b)

$$
K^{\prime \prime}=12
$$

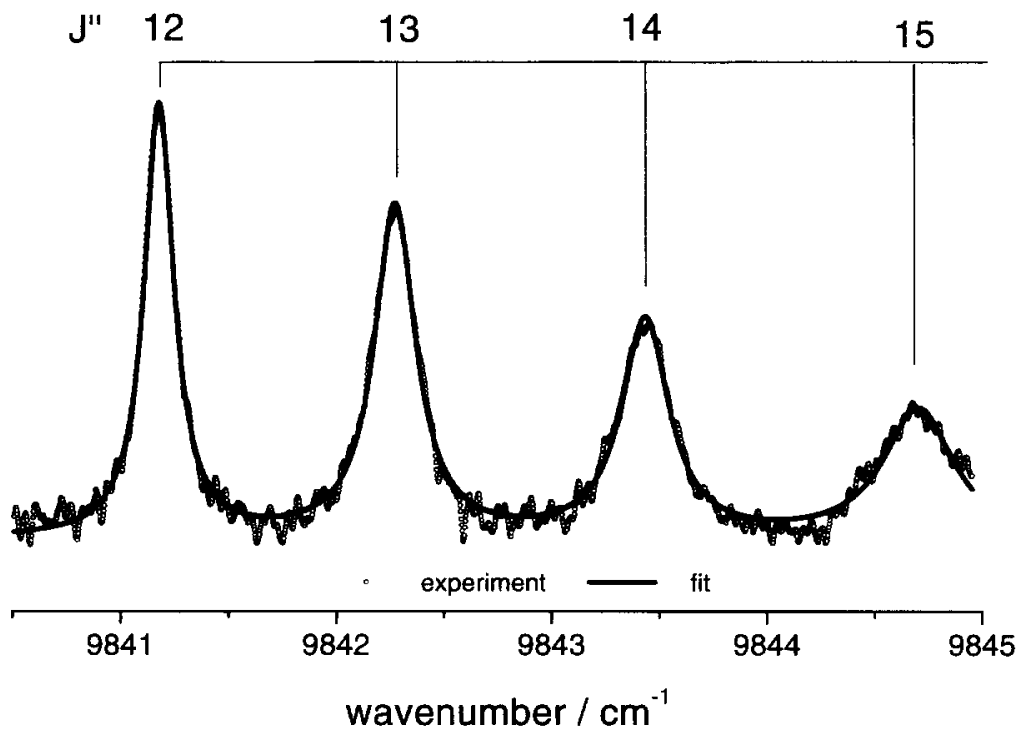

Fig. 2. (a) Typical section of the ozone spectrum around $9842 \mathrm{~cm}^{-1}$ (" $K^{\prime \prime}=12$ band") of the ${ }^{3} \mathrm{~A}_{2}(010) \leftarrow$ 'A $\mathrm{A}_{1}(000)$ transition with selection rules $\Delta K=\Delta J=-1$. Assignments for $K^{\prime \prime}$ and $J^{\prime \prime}$ are indicated. Total sample pressure $p_{\text {tot }}=45 \mathrm{mbar}$. (b) Spectrum as in (a) but with a multi-Lorentz fit (4 line profiles) superimposed. 
a)

$$
K^{\prime \prime}=8
$$

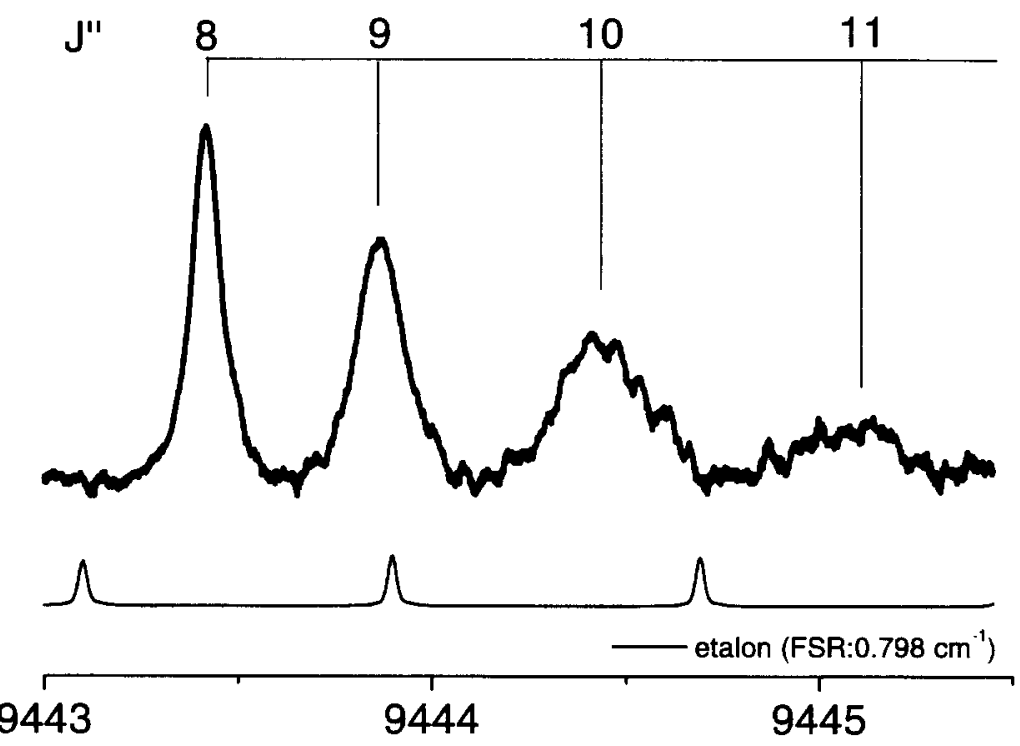

wavenumber $/ \mathrm{cm}^{-1}$

b)

$$
K^{\prime \prime}=8
$$

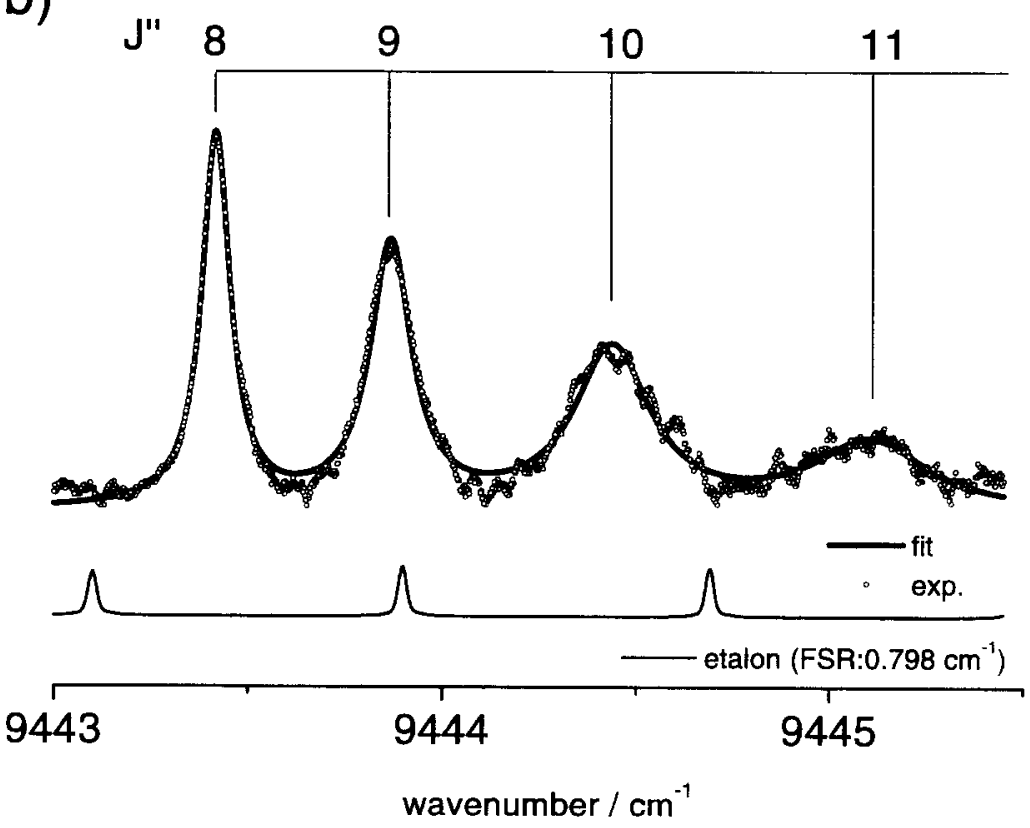

Fig. 3. (a) Typical section of the ozone spectrum around $9444 \mathrm{~cm}^{-1}$ (“ $K^{\prime \prime}=8$ band”) of the ${ }^{3} \mathrm{~A}_{2}(000) \leftarrow{ }^{\prime} \mathrm{A}_{1}(000)$ transition with selection rules $\Delta K=\Delta J=-1$. Assignments for $K^{\prime \prime}$ and $J^{\prime \prime}$ are indicated. $p_{\text {tot }}=95$ mbar. Absolute wavelength calibration: $\pm 1 \mathrm{~cm}^{-1} .(\mathrm{b})$ Spectrum as in (a) but with a multi-Lorentz fit (4 line profiles) superimposed. The etalon trace from the experiment (FSR of the etalon is $0.798 \mathrm{~cm}^{-1}$ ) is also shown. 
(Innova 200, Coherent). The broad band output of the lasers was dispersed in a high resolution homebuilt autocollimating grating spectograph with a resolving power (resolution) in triple diffraction of $0.014 \mathrm{~cm}^{-1}$ (see Fig. 1b) in the near infrared, and recorded with a 3648 element CCD array (Toshiba TCD 1301D) in the focal plane of the spectrometer's exit. Typically 2000 spectra were accumulated in order to improve the signal-to-noise ratio. The generation time (and in turn the absorption path length) of the Ti:sapphire (Nd:glass) laser was controlled by a mechanical chopper and a synchronized acousto-optic deflector (Isomet Model 1205C-2) for the time resolved measurements. Both devices defined $t_{\text {start }}$ and $t_{\text {end }}$ and thus the generation time $t_{\mathrm{g}}=t_{\text {end }}-t_{\text {start }}$. The effective absorption path could be determined simply by multiplying $t_{\mathrm{g}}$ with the speed of light $c$ and a factor $r$ [19]. The occupation ratio $r$ defined as the ratio of the intracavity absorption cell length to the total optical laser cavity length $\left(r=L_{\mathrm{abs}} / L_{\text {cav }}\right)$ was about 0.3 in these experiments. In the case of the Ti:sapphire laser medium generation times of 30-100 $\mu s$ (effective absorption path 5-10 km) and for the $\mathrm{Nd}$ :glass spectrometer generation times of about 1 ms (effective absorption path $\leq 100 \mathrm{~km}$ ) have been used. Note that the ICLA spectrometers gain medium compensates any broad band losses such that mirror reflectivities or even window deposits etc. do not play a role. For details we refer the reader to Ref. [19]. The absorption cell with the gas mixture $\left(\mathrm{O}_{3} / \mathrm{O}_{2}\right)$ was placed within the laser resonator of the ICLA spectrometer. The spectra were recorded typically at a total pressure of 10-95 mbar with ozone concentrations of $20-50 \%$. The sample pressures were measured by an MKS baratron. Ozone concentrations were determined by recording UV absorption spectra of the gas mixture. The measured spectra were frequency calibrated using an intracavity fused silica etalon and water vapor lines. We estimate the overall precision of the etalon calibration to be better than $\pm 0.02 \mathrm{~cm}^{-1}$.

\section{Results and discussion}

We have recorded high resolution absorption spectra of the first two members of the Wulf (vibrational $\nu_{2}$ bending) progression with origins at 9557 \pm 2 and $10087 \pm 2 \mathrm{~cm}^{-1}$. Our preliminary analysis of the ${ }^{3} A_{2}(000) \leftarrow{ }^{1} A_{1}(000)$ and ${ }^{3} A_{2}(010) \leftarrow{ }^{1} A_{1}(000)$ bands is mostly consistent with the analysis of Bouvier et al. [11], therefore we will use their assignments here. Following Bouvier et al. the spectrum can be partially assigned in the least congested region assuming the upper state to be ${ }^{3} \mathrm{~A}_{2}$ and assuming that the dominant transition moment involves the $x$-axis spin sublevel. The least congested parts of the spectrum (red wings) can therefore be regarded to consist mostly of $\mathrm{B}_{1} \leftarrow \mathrm{A}_{1}$ type transitions with $\Delta K$ $=\Delta J=-1$ and can, in principle, be assigned straightforwardly. From such an analysis the rotational constants, and in turn the geometry of the excited state $\left({ }^{3} \mathrm{~A}_{2}\right)$ can be derived and compared with theory [21]. A full analysis of the spectra using the formalism for singlet-triplet transitions worked out by Hougen [22] is in progress in our lab and will be reported elsewhere [23]. Although, we adopted the assignments of Bouvier et al. here we want to emphasize that all the bands are more or less strongly perturbed indicating interesting excited state (intramolecular) dynamics, an observation which has not been reported in Ref. [11]. In this letter we will entirely focus on the linewidths of the transitions in the ${ }^{3} \mathrm{~A}_{2}(000) \leftarrow{ }^{\mathrm{H}} \mathrm{A}_{1}(000)$ and ${ }^{3} \mathrm{~A}_{2}(010) \leftarrow{ }^{\mathrm{I}} \mathrm{A}_{1}(000)$ bands because they may be of considerable interest for the ozone (recombination) kinetics and for atmospheric chemistry. When we measured the above mentioned bands with a higher resolution (FWHM: $0.014 \mathrm{~cm}^{-1}$ ) and with a higher sensitivity which permitted us to record the spectra with less pressure broadening we found all lines more or less lifetime broadened (exceeding pressure and Doppler broadening considerably) indicating a strong coupling to predissociative states (pressure broadening: $<0.017$ $\mathrm{cm}^{-1}$ [24], Doppler: $0.018 \mathrm{~cm}^{-1}$, resolution: 0.014 $\mathrm{cm}^{-1}$, from a convolution of pressure and Doppler broadened lines with the apparatus function we had expected effective linewidths of $\leq 0.04 \mathrm{~cm}^{-1}$ for unperturbed lines). Fig. 2a and Fig. 3a give representative parts of the assigned $K^{\prime \prime}=12$ and $K^{\prime \prime}=8$ bands of the ${ }^{3} A_{2}(010) \leftarrow{ }^{1} A_{1}(000)$ (Fig. 2a) and ${ }^{3} \mathrm{~A}_{2}(000) \leftarrow{ }^{1} \mathrm{~A}_{1}(000)$ (Fig. 3a) transitions both with $\Delta K=\Delta J=-1$ selection rules. Moreover, the successive lines of the $K$-bands are increasingly broadened with increasing $J$. We have found this $J$-dependent (rotationally induced) broadening of the lines 


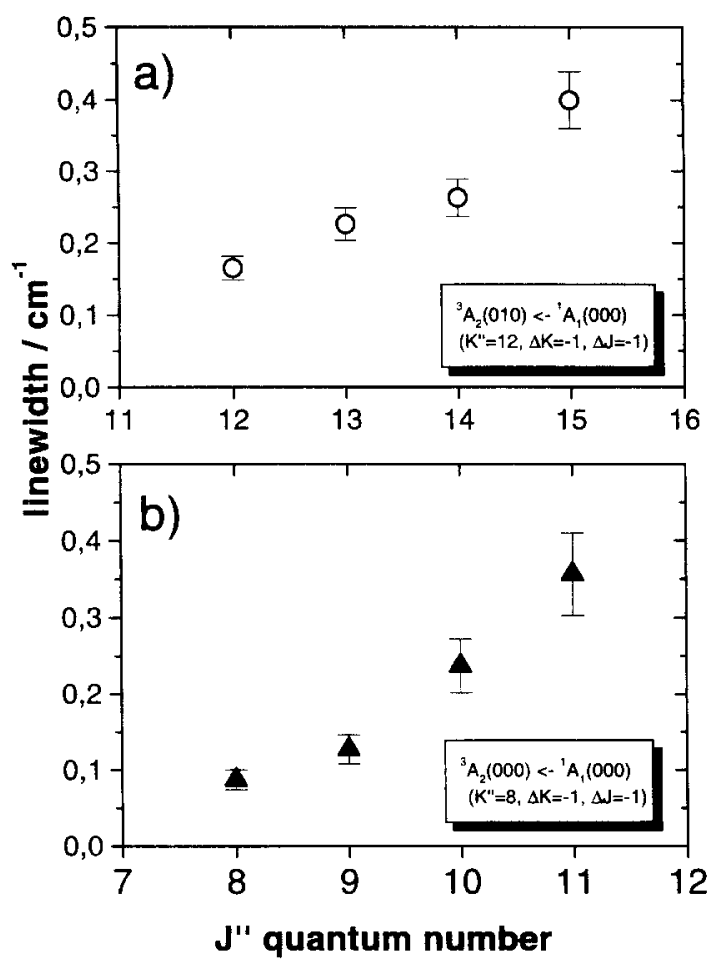

Fig. 4. Linewidths as a function of $J^{\prime \prime}\left(J^{\prime}=J^{\prime \prime}-1\right)$ for the transitions shown in Figs. 2 and 3. For more details see Figs. 3 and 4 and the text.

(also depending on $K$ ) in every resolved $K$-band in both $1 \leftarrow 0$ and $O \leftarrow O$ transitions. It should be pointed out that Bouvier et al. did not report any evidence for (progressive) line broadening other than Doppler and pressure broadening (experimental conditions: pressure broadening in their system: 0.04$0.05 \mathrm{~cm}^{-1}$, Doppler broadening: $0.02 \mathrm{~cm}^{-1}$, spectrometer resolution: $0.05 \mathrm{~cm}^{-1}$, effective linewidth: $0.07-0.08 \mathrm{~cm}^{-1}$ ). They actually estimated the radiative lifetime $\tau$ for the ${ }^{3} \mathrm{~A}_{2}$ state from the integrated (line) absorption cross sections for isolated lines and their associated Einstein coefficients to be of the order of $0.01-0.1 \mathrm{~s}$ [11] which appeared to be in agreement with calculated lifetimes [8]. From this estimate they conclude that the state is radiatively metastable. We more or less agree with the findings of Bouvier et al. [11] that the narrowest lines in the spectrum have a width close to $0.085 \mathrm{~cm}^{-1}$ (see e.g. Fig. 4), but we would like to emphasize that this is the case only for the very first members of all $K$-bands $(J=K)$. Many of these have widths larger than $0.1 \mathrm{~cm}^{-1}$ [23]. All other lines with $J>K$ are considerably broadened (observable up to $0.5 \mathrm{~cm}^{-1}$ ). We tentatively attribute the increasing linewidths with increasing $J$ to a spin-rotation and spin-orbit coupling to a perturber state which is predissociative, most likely to the electronic ground state ${ }^{\prime} A_{l}$. This conclusion is supported by our preliminary analysis of the perturbations of the corresponding singlet-triplet spectra [23]. The lineshapes have in turn been fitted to Lorentzians, as can be inspected in Figs. $2 b$ and $3 \mathrm{~b}$. The linewidths as a function of $J^{\prime \prime}$ quantum number are displayed in Fig. $4 a$ and $b$ for both $K$-bands above $\left(J^{\prime}=J^{\prime \prime}-1\right)$. Note that these increasing linewidths correlate with a drop-off (decrease) of the intensities in the spectrum. We conclude from these results that the upper state in these transitions, which is the ${ }^{3} \mathrm{~A}_{2}$, the lowest triplet state in ozone, is not metastable with regard to the lifetimes of the observed $J, K$ levels of this excited triplet state. It obviously has resolvable (but no sharp) rotational structure for $\nu_{2}^{\prime}=0,1$ (excited state bending mode) which implies that the state lives for many vibrational periods but the considerable line broadening tells us that the state does not live longer than $\approx 50-100$ ps. Actually, most of the rovibrational states of ${ }^{3} \mathrm{~A}_{2}$ live much less than $50 \mathrm{ps.} \mathrm{At} \mathrm{the}$ same time the real radiative lifetime for the perturbed states cannot be larger than about 50-100 ps because the molecules dissociate before they emit a photon. It is not clear why Bouvier et al. did not report or observe line broadening in their spectra. The signal-to-noise ratio, pressure broadening, and/or resolution may be the reason for the discrepancy in the linewidth measurements (we at the same time confirm all other spectral features and assignments of their recent work). However, by visual inspection of the published spectra also the linewidths in the inset of Fig. 2 in Ref. [11] appeared larger to us than $0.08 \mathrm{~cm}^{-1}$ (with inceasing linewidths for increasing $J^{\prime}$ ). This is not the $J, K$ progression in the $1 \leftarrow 0$ band we report here but such an effect would be in decent accord with the findings reported here and with our recent measurements [23]. In addition, we would like to stress at this point that intracavity laser absorption spectroscopy has turned out to be a powerful technique for linewidth and lineshape measurements in order to determine pressure broadening and excited state lifetimes from 


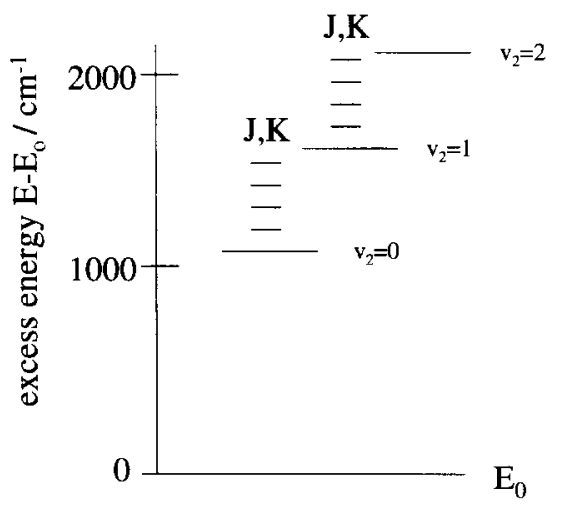

Fig. 5. Schematic energy diagram with the dissociation energy of the ground state dissociating to the products $\mathrm{O}_{2}\left({ }^{3} \Sigma_{\mathrm{g}}^{-}, \nu=0\right)+$ $O\left({ }^{3} \mathrm{P}\right)$ as a reference (in agreement with Refs. [9-11,18]). The origins of the vibrationally excited triplet state ${ }^{3} \mathrm{~A}_{2}$ with $\nu_{2}=0,1$, 2 and $J, K$ rotational levels for $\nu_{2}=0,1$ in the excited ${ }^{3} \mathrm{~A}_{2}$ state are indicated.

linewidths $[25,26]$. In the experiments we have measured the bands with a spectral resolution which would resolve Doppler and pressure broadening and with a high sensitivity allowing us to keep pressure broadening contributions as low as possible. The line broadening from Doppler and pressure broadening should be well below $0.04 \mathrm{~cm}^{-1}$, which cannot explain the measured larger widths of the lines.

In Fig. 5 we have displayed the energetic position of the origin and the rovibrational levels of the ${ }^{3} \mathrm{~A}_{2}$ state in order to discuss briefly the role of the lowest excited triplet state for the kinetics (atmospheric chemistry) and photochemistry of ozone. The origins of the ${ }^{3} B_{2}$ and ${ }^{3} B_{1}$ states in this diagram would be located considerably higher in energy at about 2000 $\mathrm{cm}^{-1}$ and $3200 \mathrm{~cm}^{-1}$, respectively, above $E_{0}=8500$ $\mathrm{cm}^{-1}$ [27]. In kinetic experiments (in thermal experiments) they would hardly be thermally accessible $\left(k_{\mathrm{B}} T=207 \mathrm{~cm}^{-1}, T=300 \mathrm{~K}\right)$ at moderate temperatures, and therefore do not play a role. The ${ }^{3} \mathrm{~A}_{2}$ state, the lowest triplet state, is also hardly accessible in thermal experiments. If so it would not play a role because its (short) lifetime (and its conjectured coupling to the ground state) would rule out a considerable impact on ozone's recombination kinetics. With regard to the photochemistry of $\mathrm{O}_{3}$ the short lifetimes also rule out metastability for this triplet state and in turn an anomalous impact on ozone concentrations in the atmosphere. The anomalies in the pressure dependence in the recombination rate of $\mathrm{O}+\mathrm{O}_{2}$ [16], and the discrepancy between $\mathrm{O}$ loss and apparent $\mathrm{O}_{3}$ formation (e.g., [12], [15]) must also come from an other source.

\section{Acknowledgements}

The authors wish to thank J. Troe for stimulating discussions and support and $U$. Wachsmuth for valuable help with the experiments. Financial support by the Deutsche Forschungsgemeinschaft (DFG) within the Sonderforschungsbereich 357 (Molekulare Mechanismen Unimolekularer Reaktionen) is gratefully acknowledged. We also enjoyed discussions on special topics of this work with $\mathrm{A}$. Kachanov and $\mathrm{H}$. Hippler.

\section{References}

[1] J.I. Steinfeld, S.M. Alder-Golden and J.W. Gallagher, J Phys. Ref. Data 16 (1987) 911, and references cited therein.

[2] O.R. Wulf, Proc. Natl. Acad. Sci (Paris) 16 (1930) 507.

[3] A. Banichevich, S. Peyerimhoff, Chem. Phys. 174 (1993) 93.

[4] A. Banichevich, S. Peyerimhoff, F. Grein, Chem. Phys. 178 (1993) 155

[5] M. Braunstein, P.J. Hay, R.L. Martin, R.T. Pack, J. Chem. Phys. 95 (1991) 8239.

[6] M. Braunstein, R.T. Pack, J. Chem. Phys. 96 (1992) 6378.

[7] M. Braunstein, R.L. Martin, P.J. Hay, J. Chem. Phys. 102 (1995) 3662.

[8] B. Minaev, H. Agren, Chem. Phys. Lett. 217 (1994) 531.

[9] S.M. Anderson, K. Mauersberger, J. Geophys. Res. 100 (1995) 3033.

[10] S. Anderson, P. Hupalo, K. Mauersberger, J. Chem. Phys. 99 (1993) 737.

[11] A.J. Bouvier, R. Bacis, B. Bussery, S. Churassy, D. Inard, M. Nota, J. Brion, J. Malicet, S.M. Anderson, Chem. Phys. Lett. 255 (1996) 263.

[12] P.G. Burton, M.D. Harvey, Nature 266 (1977) 826.

[13] C.W. von Rosenberg Jr., D.W. Trainor, J. Chem. Phys. 63 (1975) 5348.

[14] J. Shi, J.R. Barker, J. Phys. Chem. 94 (1990) 8390.

[15] N. Swanson, R.J. Celotta, Phys. Rev. Lett. 35 (1975) 783.

[16] D. Arnold, C. Xu, E.H. Kim, D. Neumark, J. Chem. Phys. 101 (1994) 912.

[17] J.R. Locker, J.A. Joens, E.J. Bair, J. Photochem. 36 (1987) 235.

[18] H. Hippler, R. Rahn and J. Troe, J. Chem. Phys. 93 (1990).

[19] B. Abel, H.H. Hamann, A.A. Kachanov and J. Troe, J. Chem. Phys. 104 (1996) 3189, and references cited therein. 
[20] T.P. Belikova, E.A. Sviridenkov, A.F. Suchkov, L.V. Titova, S.S. Churilov, JETP 35 (6) (1972) 1076.

[21] G. Herzberg, Infrared and Raman Spectra (Van Nostrand Reinhold, Princeton, NJ, 1945).

[22] J.T. Hougen, Can. J. Phys. 42 (1964) 433.

[23] B. Abel, A. Charvat, S.F. Deppe, J. Troe and U. Wachsmuth, to be published.
[24] C.C. Flannery, J.I. Steinfeld, J. Chem. Phys. 96 (1992) 8157.

[25] M. Chenevier, M.A. Melieres, F. Stoeckel, Opt. Commun. 45 (1983) 385.

[26] K. Singh, J.J. O'Brien, Quant. Spectrosc. Radiat. Trans. 52 (1994) 75

[27] A. Kailan, S. Jahn, Z. Anorg. Chem. 68 (1910) 243. 